\title{
Singular values of some modular functions*
}

\author{
November 8, 2018
}

Noburo Ishil and Maho Kobayashi

\section{Introduction}

For a positive integer $N$, let $\Gamma_{0}(N)$ and $\Gamma_{1}(N)$ be the subgroups of $\mathrm{SL}_{2}(\mathbf{Z})$ defined by

$$
\begin{aligned}
& \Gamma_{0}(N)=\left\{\left(\begin{array}{ll}
a & b \\
c & d
\end{array}\right) \in \mathrm{SL}_{2}(\mathbf{Z}) \mid c \equiv 0 \bmod N\right\} \\
& \Gamma_{1}(N)=\left\{\left(\begin{array}{ll}
a & b \\
c & d
\end{array}\right) \in \mathrm{SL}_{2}(\mathbf{Z}) \mid a-1 \equiv c \equiv 0 \bmod N\right\} .
\end{aligned}
$$

We denote by $A_{1}(N)$ and $A_{0}(N)$ the modular function fields with respect to $\Gamma_{1}(N)$ and $\Gamma_{0}(N)$ respectively. Let $\mathfrak{E}$ be a set of triples of integers $\mathfrak{a}=$ $\left[a_{1}, a_{2}, a_{3}\right]$ with the properties $0<a_{i} \leq N / 2$ and $a_{i} \neq a_{j}$ for $i \neq j$. For an element $\tau$ of complex upper half plane $\mathfrak{H}$, we denote by $L_{\tau}$ the lattice in $\mathbf{C}$ generated by 1 and $\tau$. Let $\wp\left(z ; L_{\tau}\right)$ be the Weierstrass $\wp$-function relative to the lattice $L_{\tau}$. For $\mathfrak{a} \in \mathfrak{E}$, consider a function $W_{\mathfrak{a}}(\tau)$ on $\mathfrak{H}$ defined by

$$
W_{\mathfrak{a}}(\tau)=\frac{\wp\left(a_{1} / N ; \tau\right)-\wp\left(a_{3} / N ; \tau\right)}{\wp\left(a_{2} / N ; \tau\right)-\wp\left(a_{3} / N ; \tau\right)} .
$$

This function is a modular function with respect to $\Gamma_{1}(N)$, referred in Chapter $18, \S 6$ of Lang [6]. He pointed out that it is interesting to investigate its

*2000 Mathematics Subject Classification 11F03,11G15 
special values at imaginary quadratic points. In [4] and [5], to construct generators of $A_{1}(N)$ and $A_{0}(N)$, we used the function $W_{\mathfrak{a}}(\tau)$ and the function $T_{\mathfrak{a}_{1}, \mathfrak{a}_{2}}(\tau)$ which is the trace of the product $W_{\mathfrak{a}_{1}} W_{\mathfrak{a}_{2}}\left(\mathfrak{a}_{i} \in \mathfrak{E}\right)$ relative to the extension $A_{1}(N) / A_{0}(N)$. Further we provided an explicit representation of the modular $j$-function $j(\tau)$ with those generators. In this article, we study the properties of singular values of $W_{\mathfrak{a}}$ and those of a function $T_{\mathfrak{A}, F}$ which is a generalization of the function $T_{\mathfrak{a}_{1}, \mathfrak{a}_{2}}$. See $\S 2$ for the precise definition of $T_{\mathfrak{A}, F}$. Our results in this article are as follows. In Theorem 3.7 and Corollary 4.6 we prove, for imaginary quadratic points $\alpha \in \mathfrak{H}$ and sets $\mathfrak{a}, \mathfrak{A}$ satisfying some conditions, that singular values $W_{\mathfrak{a}}(\alpha)$ are units of the ray class field $\mathfrak{K}_{N}$ modulo $N$ over $K$ and that singular values $T_{\mathfrak{A}, F}(\alpha)$ are algebraic integers in $\mathfrak{K}_{N}$. In particular, consider the triples $\mathfrak{a}_{1}=[2,3,1]$ and $\mathfrak{a}_{2}=[2,5,1]$. Then we prove in Theorem 4.4 that $W_{\mathfrak{a}_{1}}(\alpha)$ and $W_{\mathfrak{a}_{2}}(\alpha)$ generate $\mathfrak{K}_{N}$ over the field $K(\exp (2 \pi i / N))$. Let $A_{0}(N)_{\mathbf{Q}}$ be the subfield of $A_{0}(N)$ consisting of modular functions with Fourier coefficients in Q. In Proposition 4.2 we show for prime numbers $N$ that $A_{0}(N)_{\mathbf{Q}}=\mathbf{Q}\left(T_{\mathfrak{a}_{1}}, T_{\mathfrak{a}_{2}}\right)=\mathbf{Q}\left(T_{\mathfrak{a}_{i}}, T_{\mathfrak{a}_{1}, \mathfrak{a}_{2}}\right)(i=1,2)$. Further put $\mathfrak{A}_{0}=\left[\mathfrak{a}_{1}, \mathfrak{a}_{2}\right]$ and $F_{0}=X_{1}^{m} X_{2}^{n}$ for non-negative integers $m$ and $n$. In Theorem 4.3, without the assumption $N$ are prime, we show that $A_{0}(N)_{\mathbf{Q}}=\mathbf{Q}\left(j, T_{\mathfrak{A}_{0}, F_{0}}\right)$. We deduce from those results that singular values of those functions generate ring class fields over $K$ (see Theorem 4.7). Finally in $\S 5$ we study class polynomials of $T_{\mathfrak{A}, F}$ with respect to $\operatorname{Schertz} N$-systems.

In the followings, for a function $f(\tau)$ and a matrix $A=\left(\begin{array}{ll}a & b \\ c & d\end{array}\right) \in \mathrm{SL}_{2}(\mathbf{Z})$, we shall denote

$$
f[A]_{2}=f\left(\frac{a \tau+b}{c \tau+d}\right)(c \tau+d)^{-2} \text { and } f \circ A=f\left(\frac{a \tau+b}{c \tau+d}\right) .
$$

\section{Modular functions $W_{\mathfrak{a}}(\tau)$ and $T_{\mathfrak{A}, F}(\tau)$}

Let $W_{\mathfrak{a}}(\tau)$ be the function defined in $\S 1$. In [4], we showed the function $W_{\mathfrak{a}}$ is a modular function with respect to $\Gamma_{1}(N)$ and it has neither zeros nor poles on $\mathfrak{H}$. Let us consider the factor group $G(N)=\Gamma_{0}(N) /\left\{ \pm E_{2}\right\} \Gamma_{1}(N)$, where $E_{2}$ is the unit matrix. Put $\mathfrak{S}_{N}=(\mathbf{Z} / N \mathbf{Z})^{\times} /\{ \pm 1\}$. Then

$$
G(N) \cong\left\{\left(\begin{array}{cc}
\lambda^{-1} & 0 \\
0 & \lambda
\end{array}\right) \mid \lambda \in \mathfrak{S}_{N}\right\}
$$


For $\lambda \in \mathfrak{S}_{N}$, let $M_{\lambda} \in \Gamma_{0}(N)$ such that $M_{\lambda} \equiv\left(\begin{array}{cc}\lambda^{-1} & 0 \\ 0 & \lambda\end{array}\right) \bmod N$. For a tuple $\mathfrak{A}=\left[\mathfrak{a}_{1}, \ldots, \mathfrak{a}_{n}\right]\left(\mathfrak{a}_{i} \in \mathfrak{E}\right)$ and a polynomial $F=F\left(X_{1}, X_{2}, \ldots, X_{n}\right)$ $\in \mathbf{Z}\left[X_{1}, X_{2}, \ldots, X_{n}\right]$, we define a function

$$
T_{\mathfrak{A}, F}(\tau)=\sum_{\lambda \in \mathfrak{S}_{N}} F\left(W_{\mathfrak{a}_{1}} \circ M_{\lambda}, \cdots, W_{\mathfrak{a}_{n}} \circ M_{\lambda}\right) .
$$

Then obviously $T_{\mathfrak{A}, F}(\tau)$ is a modular function with respect to $\Gamma_{0}(N)$ and has no poles on $\mathfrak{H}$. For $\lambda \in \mathfrak{S}_{N}, \mathfrak{a}=\left[a_{1}, a_{2}, a_{3}\right] \in \mathfrak{E}$, define an element $\lambda \mathfrak{a}$ of $\mathfrak{E}$ by

$$
\lambda \mathfrak{a}=\left[\left\{\lambda a_{1}\right\},\left\{\lambda a_{2}\right\},\left\{\lambda a_{3}\right\}\right],
$$

where $\left\{\lambda a_{i}\right\}$ is the integer such that $\left\{\lambda a_{i}\right\} \equiv \pm \lambda a_{i} \bmod N, 0<\left\{\lambda a_{i}\right\} \leq \frac{N}{2}$.

Proposition 2.1. $\quad$ (i) $W_{\mathfrak{a}}\left(M_{\lambda} \tau\right)=W_{\lambda \mathfrak{a}}(\tau)$.

(ii) $T_{\mathfrak{A}, F}(\tau)=\sum_{\lambda \in \mathfrak{S}_{N}} F\left(W_{\lambda \mathfrak{a}_{1}}(\tau), \cdots, W_{\lambda \mathfrak{a}_{n}}(\tau)\right)$.

Proof. The assertion (i) is showed in $\S 2$ of [4]. The assertion (ii) is obvious from (i).

We denote by $T_{\mathfrak{a}}$ and $T_{\mathfrak{a}_{1}, \mathfrak{a}_{2}}$ the function $T_{\mathfrak{A}, F}$ with $\mathfrak{A}=[\mathfrak{a}], F=X_{1}$ and $\mathfrak{A}=\left[\mathfrak{a}_{1}, \mathfrak{a}_{2}\right], F=X_{1} X_{2}$ respectively.

\section{Modular equations}

Let $j$ be the modular $j$-function. Let $\Gamma$ be a subgroup of $\mathrm{SL}_{2}(\mathbb{Z})$ of finite index. For a modular function $f$ with respect to $\Gamma$, we define the modular equation of $f$ relative to $j$ by

$$
\Phi[f](X, j)=\prod_{B}(X-f \circ B),
$$

where $B$ runs over a transversal of the coset decomposition of $\mathrm{SL}_{2}(\mathbf{Z})$ by $\Gamma$. Obviously the coefficients of $\Phi[f](X, j)$ with respect to $X$ are in $\mathbf{C}(j)$. If $f$ has no poles on $\mathfrak{H}$, then the coefficients of $\Phi[f](X, j)$ are polynomials of $j$. Hereafter to avoid tedious notation, we denote by $\Phi_{\mathfrak{A}, F}(X, j)$ the equation $\Phi\left[T_{\mathfrak{A}, F}\right](X, j)$. Since $W_{\mathfrak{a}}$ and $T_{\mathfrak{A}, F}$ have no poles on $\mathfrak{H}$, we have 
$\Phi\left[W_{\mathfrak{a}}\right](X, j), \Phi_{\mathfrak{Q}, F}(X, j) \in \mathbf{C}[j][X]$. We shall show that $\Phi\left[W_{\mathfrak{a}}\right](X, j)$ and $\Phi_{\mathfrak{A}, F}(X, j) \in \mathbf{Z}[j][X]$ under some conditions imposed on $N$ and $\mathfrak{A}$. For a positive divisor $t$ of $N$, let $\Theta_{t}$ be a set of $\varphi((t, N / t))$ pairs of integers $(u, v)$ such that $(u, t)=1, u v \equiv 1 \bmod t$ and $\mathrm{u}$ are inequivalent to each other modulo $(t, N / t)$. For $(u, v) \in \Theta_{t}$ and $k \in \mathbf{Z}$, consider a matrix in $\mathrm{SL}_{2}(\mathbf{Z})$

$$
B(t, u, v, k)=\left(\begin{array}{cc}
u & (u v-1) / t+u k \\
t & v+t k
\end{array}\right) .
$$

We denote by $\mathfrak{M}_{\Theta_{t}}$ the set of matrices

$$
\left\{B(t, u, v, k) \mid(u, v) \in \Theta_{t}, k \bmod N /\left(t^{2}, N\right)\right\} .
$$

Lemma 3.1. (i) The set of matrices $\cup \mathfrak{M}_{t \mid N}$ is a transversal of the coset decomposition of $\mathrm{SL}_{2}(\mathbf{Z})$ by $\Gamma_{0}(N)$.

(ii) The set of matrices $\left\{M_{\lambda} B \mid \lambda \in \mathfrak{S}_{N}, B \in \underset{t \mid N}{\cup} \mathfrak{M}_{\Theta_{t}}\right\}$ is a transversal of the coset decomposition of $\mathrm{SL}_{2}(\mathbf{Z})$ by $\Gamma_{1}(N)\left\{ \pm E_{2}\right\}$.

Proof. The number of elements of the set is $\sum_{t \mid N} \frac{N}{\left(t^{2}, N\right)} \varphi((t, N / t))$. This is equal to $\left[\mathrm{SL}_{2}(\mathbf{Z}): \Gamma_{0}(N)\right]$ (see Exercises 11.9 [1]). It is easy to see that any distinct matrices in the set $\cup \mathfrak{M}_{t \mid N}$ are not in the same coset. Thus we have (i). The assertion (ii) is obvious from (i).

Let $\ell_{t}$ be an integer prime to $t$ and $\ell_{t}^{*}$ an integer such that $\ell_{t} \ell_{t}^{*} \equiv 1 \bmod t$. For the set $\Theta_{t}$, put

$$
\ell_{t} \Theta_{t}=\left\{\left(\ell_{t}^{*} u, \ell_{t} v\right) \mid(u, v) \in \Theta_{t}\right\} .
$$

Then obviously the set of matrices $\cup \mathfrak{M}_{t \mid N}$ is $\mathfrak{\ell}_{t} \Theta_{t}$ also a transversal of the coset decomposition. For an integer $s$ not congruent to $0 \bmod N$, let

$$
\phi_{s}(\tau)=\frac{1}{(2 \pi i)^{2}} \wp\left(\frac{s}{N} ; L_{\tau}\right)-1 / 12 .
$$

Put $q=\exp (2 \pi i \tau / N)$ and $\zeta=\exp (2 \pi i / N)$. To consider the $q$-expansion of the function $\phi_{s}[B(t, u, v, k)]_{2}$, for an integer $s$, we define two integers $\{s\}$ and 
$\mu(s)$ by the following conditions:

$$
\begin{aligned}
& 0 \leq\{s\} \leq \frac{N}{2}, \quad \mu(s)= \pm 1, \\
& \begin{cases}\mu(s)=1 & \text { if } s \equiv 0, N / 2 \bmod N \\
s \equiv \mu(s)\{s\} \quad \bmod N & \text { otherwise. }\end{cases}
\end{aligned}
$$

By Lemma 1 of [4], we have, with $s^{*}=\mu(s t) s(v+t k)$,

$$
\phi_{s}[B(t, u, v, k)]_{2}=
$$

$$
\begin{cases}\frac{\zeta^{s^{*}}}{\left(1-\zeta^{s^{*}}\right)^{2}}-\sum_{m=1}^{\infty} \sum_{n=1}^{\infty} n\left(1-\zeta^{s^{*} n}\right)\left(1-\zeta^{-s^{*} n}\right) q^{m n N} & \text { if }\{s t\}=0 \\ \sum_{n=1}^{\infty} n \zeta^{s^{*} n} q^{\{s t\} n} & \\ -\sum_{m=1}^{\infty} \sum_{n=1}^{\infty} n\left(1-\zeta^{s^{*}} q^{\{s t\} n}\right)\left(1-\zeta^{-s^{*} n} q^{-\{s t\} n}\right) q^{m n N} & \text { otherwise. }\end{cases}
$$

In particular we note the function $\phi_{s}[B(t, u, v, k)]_{2} \in \mathbf{Q}(\zeta)[[q]]$.

For an integer $\ell$ prime to $N$, let $\sigma_{\ell}$ be the automorphism of $\mathbf{Q}(\zeta)$ over $\mathbf{Q}$ defined by $\zeta^{\sigma_{\ell}}=\zeta^{\ell}$. On a function $f=\sum_{m} a_{m} q^{m}$ with $a_{m} \in \mathbf{Q}(\zeta), \sigma_{\ell}$ acts by $f^{\sigma_{\ell}}=\sum_{m} a_{m}^{\sigma_{\ell}} q^{m}$.

Lemma 3.2. Let $\ell$ be an integer prime to $N$ and $\ell^{*}$ an integer such that $\ell \ell^{*} \equiv 1 \bmod N$. Then for $(u, v) \in \Theta_{t}$ and $k \in \mathbf{Z}$,

$$
\phi_{s}[B(t, u, v, k)]_{2}^{\sigma_{\ell}}= \begin{cases}\phi_{l s}[B(t, u, v, k)]_{2} & \text { if }\{s t\}=0 \\ \phi_{s}\left[B\left(t, \ell^{*} u, \ell v, \ell k\right)\right]_{2} & \text { if }\{s t\} \neq 0\end{cases}
$$

Proof. The $q$-expansion of $\phi_{s}[B(t, u, v, k)]_{2}^{\sigma_{\ell}}$ is given by substituting $s^{*}$ by $\ell s^{*}$ in the equation (1). If $\{s t\}=0$, then we see $\ell s^{*}=(\ell s)^{*}$. If $\{s t\} \neq$ 0 , then $\ell s^{*}=\mu(s t) \ell s(v+t k)=\mu(s t) s(\ell v+\ell t k)$. By comparing the $q$ expansion of $\phi_{l s}[B(t, u, v, k)]_{2}$ or $\phi_{s}\left[B\left(t, \ell^{*} u, \ell v, \ell k\right)\right]_{2}$ in each case, we have our assertion. 
We consider two subsets $\mathfrak{E}_{1}$ and $\mathfrak{E}_{2}$ of $\mathfrak{E}$ given by

$$
\begin{aligned}
& \mathfrak{E}_{1}=\left\{\mathfrak{a} \in \mathfrak{E} \mid\left(a_{1} a_{2} a_{3}, N\right)=1\right\}, \\
& \mathfrak{E}_{2}=\left\{\mathfrak{a} \in \mathfrak{E}_{1} \mid\left(a_{i} \pm a_{3}, N\right)=1 \text { for } i=1,2\right\} .
\end{aligned}
$$

It is noted $\mathfrak{E}_{1} \neq \emptyset$ for $N \geq 7$ (resp.10) if $N$ is odd (resp.even) and $\mathfrak{E}_{2} \neq \emptyset$ for $N$ such that $(N, 6)=1, N \geq 7$. Further if $N$ is a prime number and $N \geq 7$, then $\mathfrak{E}_{1}=\mathfrak{E}_{2}=\mathfrak{E}$.

Example 3.3. Let $\mathfrak{a}_{1}=[2,3,1], \mathfrak{a}_{2}=[2,5,1], \mathfrak{a}_{3}=[1,(N-3) / 2,(N-1) / 2]$. If $N$ is a positive integer such that $(N, 6)=1, N \geq 7$. Then $\mathfrak{a}_{1}, \mathfrak{a}_{3} \in \mathfrak{E}_{2}$. Further if $(N, 30)=1$, then $\mathfrak{a}_{2} \in \mathfrak{E}_{2}$. The functions $T_{\mathfrak{a}_{i}}$ and $T_{\mathfrak{a}_{1}, \mathfrak{a}_{2}}$ are not constant. See Proposition 4.2.

Proposition 3.4. Let $\ell$ be an integer prime to $N$ and $\ell^{*}$ an integer such that $\ell \ell^{*} \equiv 1 \bmod N$. Further let $(u, v) \in \Theta_{t}$ and $k \in \mathbf{Z}$.

(i) For $\mathfrak{a}=\left[a_{1}, a_{2}, a_{3}\right] \in \mathfrak{E}_{1}$, we have

$$
\left(W_{\mathfrak{a}} \circ B(t, u, v, k)\right)^{\sigma_{\ell}}= \begin{cases}W_{\ell \mathfrak{a}} \circ B(t, u, v, k) & \text { if } t=N, \\ W_{\mathfrak{a}} \circ B\left(t, \ell^{*} u, \ell v, \ell k\right) & \text { if } t \neq N,\end{cases}
$$

where $\ell \mathfrak{a}=\left[\left\{\ell a_{1}\right\},\left\{\ell a_{2}\right\},\left\{\ell a_{3}\right\}\right]$.

(ii) For a tuple $\mathfrak{A}=\left[\mathfrak{a}_{1}, \ldots, \mathfrak{a}_{n}\right]\left(\mathfrak{a}_{i} \in \mathfrak{E}_{1}\right)$, we have

$$
\left(T_{\mathfrak{A}, F} \circ B(t, u, v, k)\right)^{\sigma_{\ell}}= \begin{cases}T_{\mathfrak{A}, F} \circ B(t, u, v, k) & \text { if } t=N, \\ T_{\mathfrak{A}, F} \circ B\left(t, \ell^{*} u, \ell v, \ell k\right) & \text { if } t \neq N .\end{cases}
$$

Proof. By definition of $W_{\mathfrak{a}}$ we have

$$
W_{\mathfrak{a}}(\tau)=\frac{\phi_{a_{1}}(\tau)-\phi_{a_{3}}(\tau)}{\phi_{a_{2}}(\tau)-\phi_{a_{3}}(\tau)}
$$

Therefore, (i) follows from Lemma 3.2 and (ii) is obvious from (i) and Proposition 2.1.

It is noted that for $t=1, N$ to obtain the results in Proposition 3.4, we do not need the condition $\mathfrak{a}_{i} \in \mathfrak{E}_{1}$. 
Proposition 3.5. For $\mathfrak{A}$ with $\mathfrak{a}_{i} \in \mathfrak{E}, T_{\mathfrak{A}, F}$ and $T_{\mathfrak{A}, F} \circ B(1,1,1,-1)$ have Fourier coefficients in $\mathbf{Q}$.

Proof. Since $B(N, u, v, k) \in \Gamma_{0}(N)$, by Proposition 3.4 (ii), $T_{\mathfrak{A}, F}^{\sigma_{\ell}}=T_{\mathfrak{A}, F}$. By the same proposition, we have $\left(T_{\mathfrak{A}, F} \circ B(1,1,1,-1)\right)^{\sigma_{\ell}}=T_{\mathfrak{A}, F} \circ B\left(1, \ell^{*}, \ell,-\ell\right)$. Since $B(1,1,1,-1) B\left(1, \ell^{*}, \ell,-\ell\right)^{-1} \in \Gamma_{0}(N)$, we see $\left(T_{\mathfrak{A}, F} \circ B(1,1,1,-1)\right)^{\sigma_{\ell}}=$ $T_{\mathfrak{A}, F} \circ B(1,1,1,-1)$.

Put

$$
\begin{array}{r}
\Phi\left[W_{\mathfrak{a}}\right](X, j)=X^{\Psi_{1}(N)}+\sum_{i=1}^{\Psi_{1}(N)} C[\mathfrak{a}]_{i} X^{\Psi_{1}(N)-i} \\
\Phi_{\mathfrak{A}, F}(X, j)=X^{\Psi_{0}(N)}+\sum_{i=1}^{\Psi_{0}(N)} C_{\mathfrak{A}, i} X^{\Psi_{0}(N)-i}
\end{array}
$$

where $\Psi_{0}(N)=\left[\mathrm{SL}_{2}(\mathbf{Z}): \Gamma_{0}(N)\right]=N \prod_{p \mid N}\left(1+\frac{1}{p}\right), \Psi_{1}(N)=\left[\mathrm{SL}_{2}(\mathbf{Z})\right.$ : $\left.\Gamma_{1}(N)\right]=\frac{\varphi(N) \Psi_{0}(N)}{2}$ and $p$ are prime divisors of $N$.

Theorem 3.6. (i) If $\mathfrak{a} \in \mathfrak{E}_{1}$, then the modular equation $\Phi\left[W_{\mathfrak{a}}\right] \in \mathbf{Q}[j][X]$. Further if $N$ is odd and $\mathfrak{a} \in \mathfrak{E}_{2}$, then $\Phi\left[W_{\mathfrak{a}}\right] \in \mathbf{Z}[j][X]$.

(ii) Let $\mathfrak{A}=\left[\mathfrak{a}_{1}, \ldots, \mathfrak{a}_{n}\right]$. If $\mathfrak{a}_{k} \in \mathfrak{E}_{1}$ for all $k$, then the modular equation $\Phi_{\mathfrak{A}, F} \in \mathbf{Q}[j][X]$. Further if $N$ is odd and $\mathfrak{a}_{k} \in \mathfrak{E}_{2}$ for all $k$, then $\Phi_{\mathfrak{A}, F} \in \mathbf{Z}[j][X]$.

Proof. We know the coefficients $C[\mathfrak{a}]_{i}, C_{\mathfrak{A}, i} \in \mathbf{Q}(\zeta)((q))$. To show (i), we have only to prove that they are invariant under the action of $\sigma_{\ell}$ for all $\ell$ prime to $N$. By (i) of Proposition 2.1, we see $W_{\mathfrak{a}} \circ\left(M_{\lambda} B\right)=W_{\lambda \mathfrak{a}} \circ B$. Thus by Proposition 3.4, we have

$$
\left(W_{\mathfrak{a}} \circ\left(M_{\lambda} B(t, u, v, k)\right)\right)^{\sigma_{\ell}}= \begin{cases}W_{\mathfrak{a}} \circ\left(M_{\bar{\ell} \lambda} B(t, u, v, k)\right) & \text { if } t=N, \\ W_{\mathfrak{a}} \circ\left(M_{\lambda} B\left(t, \ell^{*} u, \ell v, \ell k\right)\right) & \text { if } t \neq N\end{cases}
$$

where $\bar{\ell}$ is the element of $\mathfrak{S}_{N}$ induced by $\ell$. Since $C[\mathfrak{a}]_{i}$ is an elementary symmetric polynomial of $W_{\mathfrak{a}} \circ\left(M_{\lambda} B(t, u, v, k)\right)$, we know that $C[\mathfrak{a}]_{i}^{\sigma_{\ell}}=C[\mathfrak{a}]_{i}$. Therefore We have $C[\mathfrak{a}]_{i} \in \mathbb{Q}[j]$. Assume that $N$ is odd. Let us consider the $q$ expansions of the function $\phi_{a}[B]_{2}-\phi_{b}[B]_{2}$ for $a, b \in \mathbf{Z},(a b(a \pm b), N)=1$ and 
$B \in \mathfrak{M}_{\Theta_{t}}$. First of all, let $t \neq N$. Then $\{a t\} \neq\{b t\}$. Let $l=\min (\{a t\},\{b t\})$. Then by (1), for an integer $s$

$$
\phi_{a}[B]_{2}(\tau)-\phi_{b}[B]_{2}= \pm \zeta^{s} q^{l}+O\left(q^{l+1}\right) \in \mathbf{Z}[\zeta][[q]] .
$$

Thus, $W_{\mathfrak{a}} \circ B \in \mathbf{Z}[\zeta]((q))$. Next we shall consider the case $t=N$. We can take $M_{\Theta_{N}}=\left\{\left(\begin{array}{ll}1 & 0 \\ N & 1\end{array}\right)\right\}$. Put $B=\left(\begin{array}{ll}1 & 0 \\ N & 1\end{array}\right)$. By $(1)$, we see

$$
\begin{aligned}
\phi_{a}[B]_{2}(\tau)- & \phi_{b}[B]_{2} \\
= & \frac{\zeta^{a}\left(1-\zeta^{b-a}\right)\left(1-\zeta^{b+a}\right)}{\left(1-\zeta^{a}\right)^{2}\left(1-\zeta^{b}\right)^{2}} \\
& -\sum_{m=1}^{\infty} \sum_{n=1}^{\infty} n\left\{\left(1-\zeta^{a n}\right)\left(1-\zeta^{-a n}\right)-\left(1-\zeta^{b n}\right)\left(1-\zeta^{-b n}\right)\right\} q^{m n N} .
\end{aligned}
$$

Let

$$
\begin{aligned}
\theta_{a, b} & =\frac{\zeta^{a}\left(1-\zeta^{b-a}\right)\left(1-\zeta^{b+a}\right)}{\left(1-\zeta^{a}\right)^{2}\left(1-\zeta^{b}\right)^{2}} \\
h(q) & =-\sum_{m=1}^{\infty} \sum_{n=1}^{\infty} n\left\{\left(1-\zeta^{a n}\right)\left(1-\zeta^{-a n}\right)-\left(1-\zeta^{b n}\right)\left(1-\zeta^{-b n}\right)\right\} q^{m n N}
\end{aligned}
$$

Then

$$
\phi_{a}[B]_{2}-\phi_{b}[B]_{2}=\theta_{a, b}\left(1-\frac{1}{\theta_{a, b}} h(q)\right)
$$

Since $\frac{1-\zeta^{s}}{1-\zeta^{r}} \in \mathbf{Z}[\zeta]^{\times}$for integers $r, s$ such that $(r s, N)=1$, we see

$$
\frac{1}{\theta_{a, b}}=\frac{\left(1-\zeta^{a}\right)\left(1-\zeta^{b}\right)}{\zeta^{a}\left(1-\zeta^{b-a}\right)\left(1-\zeta^{b+a}\right)}\left(1-\zeta^{a}\right)\left(1-\zeta^{b}\right) \in \mathbf{Z}[\zeta] .
$$

Therefore for some $h(q), f(q) \in \mathbf{Z}[\zeta][[q]]$

$$
W_{\mathfrak{a}} \circ B=\frac{\theta_{a_{1}, a_{3}}(1-h(q))}{\theta_{a_{2}, a_{3}}(1-f(q))}=\frac{\theta_{a_{1,3}}}{\theta_{a_{2}, 3}}\left(1+f(q)+f(q)^{2}+\cdots\right)(1-h(q)) .
$$

Since

$$
\frac{\theta_{a_{1,3}}}{\theta_{a_{2}, 3}}=\frac{\zeta^{a_{1}}}{\zeta^{a_{2}}}\left(\frac{1-\zeta^{a_{2}}}{1-\zeta^{a_{1}}}\right)^{2} \frac{\left(1-\zeta^{a_{3}-a_{1}}\right)\left(1-\zeta^{a_{3}+a_{1}}\right)}{\left(1-\zeta^{a_{3}-a_{2}}\right)\left(1-\zeta^{a_{3}+a_{2}}\right)} \in \mathbf{Z}[\zeta]^{\times},
$$


$W_{\mathfrak{a}} \circ B \in \mathbf{Z}[\zeta][[q]]$. Therefore by (i) of Proposition 2.1, we have $W_{\mathfrak{a}} \circ\left(M_{\lambda} B\right) \in$ $\mathbf{Z}[\zeta]((q))$ for all $\lambda \in \mathfrak{S}_{n}$ and $B \in \cup_{t \mid N} \Theta_{t}$. Thus $C[\mathfrak{a}]_{i} \in \mathbf{Z}[\zeta]((q))$. By applying the above argument, we have $C[\mathfrak{a}]_{i} \in \mathbf{Z}[j]$. This shows (i). Next we shall prove (ii). By (ii) of Proposition [3.4, we have

$$
\left\{\left(T_{\mathfrak{A}, F} \circ B\right)^{\sigma_{\ell}} \mid B \in \mathfrak{M}_{\Theta_{t}}\right\}=\left\{T_{\mathfrak{A}, F} \circ B \mid B \in \mathfrak{M}_{\ell \Theta_{t}}\right\} .
$$

Since $\underset{t \mid N}{\cup} \mathfrak{M}_{\ell \Theta_{t}}$ is a transversal of coset decomposition of $\operatorname{SL}_{2}(\mathbf{Z})$ by $\Gamma_{0}(N)$, we obtain $C_{\mathfrak{A}, i}^{\sigma_{\ell}}=C_{\mathfrak{A}, i}$. This shows $C_{\mathfrak{A}, i} \in \mathbf{Q}[j]$. If $N$ is odd and $\mathfrak{a} \in \mathfrak{E}_{2}, \lambda \in$ $\mathfrak{S}_{N}$, then $\lambda \mathfrak{a} \in \mathfrak{E}_{2}$. Proposition 2.1 shows $T_{\mathfrak{A}, F} \circ B \in \mathbf{Z}[\zeta]((q))$. Therefore $C_{\mathfrak{A}, i} \in \mathbf{Z}[\zeta]((q))$. Since $C_{\mathfrak{A}, i}^{\sigma_{\ell}}=C_{\mathfrak{A}, i}$, this shows $C_{\mathfrak{A}, i} \in \mathbf{Z}[j]$.

Let $K$ be an imaginary quadratic field and $\mathfrak{K}_{N}$ the ray class field modulo $N$ over $K$.

Theorem 3.7. Assume that $N$ is odd. Let $\alpha$ be an element of $\mathfrak{H}$ such that $K=\mathbf{Q}(\alpha)$.

(i) If $\mathfrak{a} \in \mathfrak{E}_{2}$, then $W_{\mathfrak{a}}(\alpha)$ is a unit of $\mathfrak{K}_{N}$.

(ii) Let $\mathfrak{A}=\left[\mathfrak{a}_{1}, \ldots, \mathfrak{a}_{n}\right]$. If $\mathfrak{a}_{k} \in \mathfrak{E}_{2}$ for all $k$, then $T_{\mathfrak{A}, F}(\alpha)$ is an algebraic integer of $\mathfrak{K}_{N}$.

Proof. By Complex multiplication theory, $j(\alpha)$ is an algebraic integer. Theorem 3.6 shows that $\Phi\left[W_{\mathfrak{a}}\right](X, j(\alpha))$ and $\Phi_{\mathfrak{A}, F}(X, j(\alpha))$ are monic polynomials with algebraic integer coefficients. Thus $W_{\mathfrak{a}}(\alpha), T_{\mathfrak{A}, F}(\alpha)$ are algebraic integers. By Corollary to Theorem 2 in $\S 10.1$ of [6], they are in $\mathfrak{K}_{N}$. Let $\mathfrak{a}^{\prime}=\left[a_{2}, a_{1}, a_{3}\right]$. Since $W_{\mathfrak{a}}^{-1}=W_{\mathfrak{a}^{\prime}}$ and $\mathfrak{a}^{\prime} \in \mathfrak{E}_{2}, W_{\mathfrak{a}}(\alpha)^{-1}$ is an algebraic integer. Hence it is a unit.

\section{Ray class field and ring class field}

Let $K$ be a subfield of $\mathbf{C}$ and $\Gamma$ a subgroup of $\mathrm{SL}_{2}(\mathbf{Z})$ of finite index. We denote by $A(\Gamma)_{K}$ the field of all modular functions with respect to $\Gamma$ having Fourier coefficients in $K$. Further put $A_{0}(N)_{K}=A\left(\Gamma_{0}(N)\right)_{K}, A_{1}(N)_{K}=$ $A\left(\Gamma_{1}(N)\right)_{K}$. Let $\zeta=\exp (2 \pi i / N)$.

Proposition 4.1. Put $\mathfrak{a}_{1}=[2,3,1], \mathfrak{a}_{2}=[2,5,1]$. If $N \geq 11, N \neq 12$, then

$$
A_{1}(N)_{\mathbf{Q}(\zeta)}=\mathbf{Q}(\zeta)\left(j, W_{\mathfrak{a}_{1}}\right)=\mathbf{Q}(\zeta)\left(j, W_{\mathfrak{a}_{2}}\right)=\mathbf{Q}(\zeta)\left(W_{\mathfrak{a}_{1}}, W_{\mathfrak{a}_{2}}\right) .
$$


Proof. The assertion is deduced from the result $A_{1}(N)_{\mathbf{C}}=\mathbf{C}\left(j, W_{\mathfrak{a}_{i}}\right)=$ $\mathbf{C}\left(W_{\mathfrak{a}_{1}}, W_{\mathfrak{a}_{2}}\right)$ and $W_{\mathfrak{a}_{i}} \in A_{1}(N)_{\mathbf{Q}(\zeta)}(i=1,2)$ in Lemma 1 and Theorems 1 and 5 of [4].

Let $m$ and $n$ be non-negative integers. Put $F=X_{1}^{m} X_{2}^{n}$ and $\mathfrak{A}=\left[\mathfrak{a}_{1}, \mathfrak{a}_{2}\right]$ with $\mathfrak{a}_{1}=[2,3,1], \mathfrak{a}_{2}=[2,5,1]$. For a while we shall consider the function $T_{\mathfrak{A}, F}$. By Theorem 3.2 of [5], for any $\mathfrak{b}=\left[b_{1}, b_{2}, b_{3}\right] \in \mathfrak{E}$, the order of the $q$-expansion of $W_{\mathfrak{b}}$ at the point $u / t$ is equal to $\min \left(\left\{t b_{1}\right\},\left\{t b_{3}\right\}\right)-$ $\min \left(\left\{t b_{2}\right\},\left\{t b_{3}\right\}\right)$. In particular, the order of $q$-expansion of $W_{\mathfrak{b}} \circ B(t, u, v, k)$ depends only on $t$ and it equals to that of $W_{\mathfrak{b}}$ at the point $1 / t$. For any integers $a, b$ and $c$, we see $\{\{a b\} c\}=\{a\{b c\}\}$. Thus the order of $q$-expansion of $W_{\lambda \mathfrak{a}_{i}} \circ B(t, u, v, k)$ is that of $W_{\mathfrak{a}_{i}}$ at the point $1 /\{\lambda t\}$. Let $\omega_{i}(\ell)$ be the order of $q$-expansion of $W_{\mathfrak{a}_{i}}$ at the point $1 / \ell$ for $\ell \in \mathbf{Z}, 1 \leq \ell \leq N / 2$. By $\S 3$ of [4], we know $\omega_{i}(\ell)<0$ if and only if $\ell>\frac{2 N}{5}$ (resp. $\frac{3 N}{7}$ ) for $i=1$ (resp. $i=2)$. We have $\omega_{i}(\ell)=(i+1) N-(2 i+3) \ell$ for $2 N / 5<\ell \leq N / 2$ and in this range, obviously $\omega_{i}(\ell)$ is a strictly decreasing function of $\ell$. Furthermore $\omega_{i}(\ell) \equiv 0 \bmod (\ell, N)$.

Proposition 4.2. Assume that $N$ is a prime number and $N>7$. Put $\mathfrak{a}_{1}=[2,3,1], \mathfrak{a}_{2}=[2,5,1]$ and $\mathfrak{a}_{3}=[1,(N-3) / 2,(N-1) / 2]$. Then for $i=1,3$ and $j=1,2,3$

$$
A_{0}(N)_{\mathbf{Q}}=\mathbf{Q}\left(T_{\mathfrak{a}_{i}}, T_{\mathfrak{a}_{2}}\right)=\mathbf{Q}\left(T_{\mathfrak{a}_{j}}, T_{\mathfrak{a}_{1}, \mathfrak{a}_{2}}\right) .
$$

Proof. Put $T_{i}=T_{\mathfrak{a}_{i}}$ for $i=1,2,3$ and $T_{4}=T_{\mathfrak{a}_{1}, \mathfrak{a}_{2}}$. Since $N$ is a prime number, the group $\Gamma_{0}(N)$ has two cusps represented by $i \infty$ and 1 . By Theorem 3.2 of [5], for any $\mathfrak{b}=\left[b_{1}, b_{2}, b_{3}\right] \in \mathfrak{E}, W_{\mathfrak{b}}$ is regular at the point $i \infty$. Therefore the functions $T_{i}(i=1, \ldots, 4)$ are regular at $i \infty$. Let us denote by $d_{i}$ the order of the pole of $T_{i}$ at the cusp 1 . We know $\omega_{i}(\lambda)$ has the smallest value only for $\lambda=(N-1) / 2$. Thus, we have $d_{1}=(N-5) / 2, d_{2}=(N-7) / 2$ and $d_{4}=N-6$. Let us determine $d_{3}$. The function $W_{\mathfrak{a}_{3}}$ has a pole of order $(N-5) / 2$ at 1 . Let $\lambda>1$. The function $W_{\lambda \mathfrak{a}_{3}}$ has a pole at 1 if $\lambda<\{\lambda(N-1) / 2\}<\{\lambda(N-3) / 2\}$ or $\lambda<\{\lambda(N-3) / 2\}<\{\lambda(N-1) / 2\}$. In the former case, the order $d_{\lambda}$ of pole of $W_{\mathfrak{a}_{3}}$ at $1 / \lambda$ is $\{\lambda(N-1) / 2\}-\lambda$. Since $\{\lambda(N-1) / 2\}<\{\lambda(N-3) / 2\}$, we know $\{\lambda(N-1) / 2\} \leq(N-3) / 2$. Thus $d_{\lambda}<(N-5) / 2$. In the latter case, $d_{\lambda}=\{\lambda(N-3) / 2\}-\lambda$. Since $\lambda>1,\{\lambda(N-3) / 2\} \leq(N-3) / 2$, we know $d_{\lambda}<(N-5) / 2$. Therefore we have $d_{3}=(N-5) / 2$. Proposition 3.5 shows that $T_{i} \in A_{0}(N)_{\mathbf{Q}}$. Since the modular curve $X_{0}(N)$ of $\Gamma_{0}(N)$ is defined over $\mathbf{Q}$, by Proposition 2.6 (a) in 
Chapter II of [9], $d_{i}=\left[A_{0}(N)_{\mathbf{Q}}: \mathbf{Q}\left(T_{i}\right)\right]$. Since $((N-5) / 2,(N-7) / 2)=1$ and $((N-5)(N-7),(N-6))=1$, we have our assertion.

Theorem 4.3. Let $m$ and $n$ be non-negative integers. Assume that $N$ does not divide $5 m+7 n(\operatorname{resp} .2(5 m+7 n))$ and $N>9$ (resp. 36) in the case $N$ is odd (resp. even). Put $\mathfrak{A}=\left[\mathfrak{a}_{1}, \mathfrak{a}_{2}\right]$ and $F=X_{1}^{m} X_{2}^{n}$. Further assume that $N \not \equiv 0 \bmod 4$ in the case $m+n$ is even. Then we have $A_{0}(N)_{\mathbf{Q}}=\mathbf{Q}\left(j, T_{\mathfrak{A}, F}\right)$.

Proof. Put $T=T_{\mathfrak{A}, F}$. By Theorem 3 of Chapter 6 of [6], the field $A(\Gamma(N))_{\mathbf{Q}(\zeta)}$ is a Galois extension over $\mathbf{Q}(j)$ with the Galois group $\mathrm{GL}_{2}(\mathbf{Z} / N \mathbf{Z}) /\left\{ \pm E_{2}\right\}$ and the field $A_{0}(N)_{\mathbf{Q}}$ is the fixed field of the subgroup $\left\{\left(\begin{array}{ll}* & * \\ 0 & *\end{array}\right)\right\} /\left\{ \pm E_{2}\right\}$. Since $T \in A_{0}(N)_{\mathbf{Q}}$, to prove the assertions, it is sufficient to show that if $T \circ A=T$ for $A \in \mathrm{SL}_{2}(\mathbf{Z})$, then $A \in \Gamma_{0}(N)$. Let us consider the transversal $\{B(t, u, v, k)\}$ of the coset decomposition of $\mathrm{SL}_{2}(\mathbf{Z})$ by $\Gamma_{0}(N)$. Let $\omega(\ell)$ be the order of $q$-expansion of $W_{\mathfrak{a}_{1}}^{m} W_{\mathfrak{a}_{2}}^{n}$ at the point $1 / \ell$. Then obviously $\omega(\ell)=m \omega_{1}(\ell)+n \omega_{2}(\ell)$. Let $t$ be a divisor of $N$. If $\lambda$ runs over $\mathfrak{S}_{N}$, then $\{\lambda t\}$ runs over all integers $u$ such that $0 \leq u \leq N / 2,(u, N)=t$. Therefore $d \geq$ $\min \{\omega(\ell) \mid 0 \leq \ell \leq N / 2,(\ell, N)=u\}$. Furthermore if $\omega(\ell)$ has the smallest value for only one $\ell$, then we have equality. Let $u_{t}$ be the greatest integer such that $\left(u_{t}, N\right)=t$ and $u_{t} \leq N / 2$. Let $t \neq N$. Assume that $T \circ B(t, u, v, k)=T$. Put $L=B(1,1,1,-1)=\left(\begin{array}{cc}1 & -1 \\ 1 & 0\end{array}\right)$. Then $T \circ(B(t, u, v, k) L)=T \circ L$. We know $B(t, u, v, k) L=\left(\begin{array}{cc}* & * \\ t(k+1)+v & -t\end{array}\right)$. Let $\delta=(t(k+1)+v, N)$. Then we can take an integer $\xi$ so that $\xi((k+1) t+v)+\delta t \equiv 0 \bmod N$ and $(\xi, \delta)=1$. For an integer $\eta$ such that $\xi \eta \equiv 1 \bmod \delta$, put $A=\left(\begin{array}{cc}\eta & (\xi \eta-1) / \delta \\ \delta & \xi\end{array}\right)$. Since $B(t, u, v, k) L A^{-1} \in \Gamma_{0}(N)$, we have $T \circ A=T \circ L$. Let $d$ be the order of $q$-expansion of $T \circ A$ and $d_{1}$ the order of $q$-expansion of $T \circ L$. In particular, the assumption implies that $d=d_{1} \equiv 0 \bmod \delta$. In the case $\delta \neq 1$, we shall show that $d \neq d_{1}$. If $N$ is even, then $u_{N / 2}=N / 2$. If $\delta \neq N / 2$, then $u_{\delta}$ is as follows. 


\begin{tabular}{|c|c|c|c|}
\hline$N \bmod 4$ & $N / \delta \bmod 4$ & $u_{1}$ & $u_{\delta}$ \\
\hline 1,3 & 1,3 & $(N-1) / 2$ & $(N-\delta) / 2$ \\
\hline 2 & 0,2 & $(N-4) / 2$ & $(N-4 \delta) / 2$ \\
\hline 2 & 1,3 & $(N-4) / 2$ & $(N-\delta) / 2$ \\
\hline 0 & 1,3 & $(N-2) / 2$ & $(N-\delta) / 2$ \\
\hline 0 & 2 & $(N-2) / 2$ & $(N-4 \delta) / 2$ \\
\hline 0 & 0 & $(N-2) / 2$ & $(N-2 \delta) / 2$ \\
\hline
\end{tabular}

If we put $u_{1}=(N-\epsilon) / 2$ with $\epsilon=1$ (resp. 2,4) in the case $N$ is odd (resp. even), we see easily $d_{1}=\omega\left(u_{1}\right)=((5 m+7 n) \epsilon-(m+n) N) / 2$ and $d \geq \min \left(0, \omega\left(u_{\delta}\right)\right)$. It is noted that our assumption implies $d_{1}<0$. If $\delta=N$, then $d \geq 0$. Thus $d \neq d_{1}$. If $\delta=N / 2$, then $d \equiv 0 \bmod N / 2$. By assumption, $d_{1} \not \equiv 0 \bmod N / 2$. This implies $d \neq d_{1}$. Let $\delta \neq 1, N / 2, N$. Except the case $N \equiv 2 \bmod 4$ and $\delta=2$, we have $u_{\delta}<u_{1}$. Thus $d \neq d_{1}$. In the exceptional case, we have $u_{\delta}>u_{1}$. Since there exists only one $\lambda$ such that $\{2 \lambda\}=u_{2}$, we have $d<d_{1}$. Let us consider the case $\delta=1$. Then $d=d_{1}$. By (1), for a matrix $M=\left(\begin{array}{ll}* & * \\ 1 & k\end{array}\right)$ of $\mathrm{SL}_{2}(\mathbf{Z})$ and $0<s \leq N / 2$, we have

$\varphi_{s} \circ M=\zeta^{s^{*}} q^{s}+\zeta^{-s^{*}} q^{N-s}+2 \zeta^{2 s^{*}} q^{2 s}+2 \zeta^{-2 s^{*}} q^{2(N-s)}-q^{N}+$ (higher terms)

where $s^{*}=\mu(s) s k=s k$. If we put $s=s_{r}=\left\{r u_{1}\right\}$ for $r=1,2,3,5$, then $s_{r}=(N-r \epsilon) / 2, s_{r}^{*}=r u_{1} k$ for $r=1,3,5$ and $s_{2}=\epsilon, s_{2}^{*}=-2 u_{1} k$. Since $(N-\epsilon) / 2>2 \epsilon$, we have

$$
\begin{aligned}
& \left(\varphi_{s_{2}}-\varphi_{s_{1}}\right) \circ M=\zeta^{-2 u_{1} k} q^{\epsilon}\left(1+2 \zeta^{-2 u_{1} k} q^{\epsilon}+O\left(q^{\epsilon+1}\right)\right), \\
& \left(\varphi_{s_{3}}-\varphi_{s_{1}}\right) \circ M=\zeta^{3 u_{1} k} q^{(N-3 \epsilon) / 2}\left(1-\zeta^{-2 u_{1} k} q^{\epsilon}+O\left(q^{\epsilon+1}\right)\right), \\
& \left(\varphi_{s_{5}}-\varphi_{s_{1}}\right) \circ M=\zeta^{5 u_{1} k} q^{(N-5 \epsilon) / 2}\left(1+O\left(q^{\epsilon+1}\right)\right),
\end{aligned}
$$

where the notation $O\left(q^{n}\right)$ denotes a $q$-series of order greater than or equal to $n$. Because the assumption for $N$ implies $d_{1}+\epsilon<0, \omega\left(u_{1}-1\right)$, we see by (2),

$$
T \circ M=\zeta^{-(5 m+7 n) u_{1} k} q^{d_{1}}\left(1+(3 m+2 n) \zeta^{-2 u_{1} k} q^{\epsilon}+O\left(q^{\epsilon+1}\right)\right) .
$$

If we compare the coefficients of $T \circ A(k=\xi)$ with those of $T \circ L(k=0)$, we see $\zeta^{-(5 m+7 n) u_{1} \xi}=\zeta^{-2 u_{1} \xi}=1$. If $(5 m+7 n)$ is odd, then, since $\left(u_{1}, N\right)=1$, we have $\xi \equiv 0 \bmod N$. Since $\xi(t(k+1)+v)+t \equiv 0 \bmod N$, we have $t \equiv 0$ 
$\bmod N$. This gives a contradiction. Obviously if $(5 m+7 n, N)=1$, we have also a contradiction. For the case $5 m+7 n$ is even, we have $\zeta^{-2 u_{1} \xi}=1$. This shows $2 t \equiv 0 \bmod N$. Therefore if $N$ is odd, we have a contradiction. Let $N \equiv 2 \bmod 4$ and $t=N / 2$. It is noted $\Theta_{N / 2}=\{B(N / 2,1,1, k) \mid k=$ $0,1\}$. Since $(N / 2,2)=1$, we can take integers $x$ and $y$ such that $(N / 2) x+$ $2 y=1$. Consider a matrix $A=\left(\begin{array}{cc}x & -1 \\ 2 y & t\end{array}\right)$ of $\mathrm{SL}_{2}(\mathbf{Z})$. It is easy to see that $B(N / 2,1,1, k) A B\left(1,1,1, k(N / 2)^{2}-1\right)^{-1}, A B(2,1,1,-y)^{-1} \in \Gamma_{0}(N)$. Therefore we have $T \circ B\left(1,1,1, k(N / 2)^{2}-1\right)=T \circ B(2,1,1,-y)$. However the above argument for $N \equiv 2 \bmod 4$ and $\delta=2$ shows the order of $q$ expansions of the functions $T \circ B\left(1,1,1, k(N / 2)^{2}-1\right)$ and $T \circ B(2,1,1,-y)$ are distinct.

Theorem 4.4. Let $\alpha \in \mathfrak{H}$ such that $\mathbf{Z}[\alpha]$ is a maximal order of $K$. Further let $\mathfrak{a}_{1}=[2,3,1]$ and $\mathfrak{a}_{2}=[2,5,1]$. If $N=11$ or $N \geq 13$, then

$$
\mathfrak{K}_{N}=K\left(\zeta, j(\alpha), W_{\mathfrak{a}_{1}}(\alpha)\right)=K\left(\zeta, j(\alpha), W_{\mathfrak{a}_{2}}(\alpha)\right)=K\left(\zeta, W_{\mathfrak{a}_{1}}(\alpha), W_{\mathfrak{a}_{2}}(\alpha)\right) .
$$

Proof. Our assertion follows from Theorems 1 and 2 of [3] and Proposition 4.1 .

For a positive integer $m$, let $O_{m}$ be the order of conductor $m$ of $K$ and $R_{m}$ the ring class field associated with the order $O_{m}$. Consider the group

$$
\Gamma^{0}(N)=\left\{\left(\begin{array}{ll}
a & b \\
c & d
\end{array}\right) \in S L(2, \mathbf{Z}) \mid b \equiv 0 \quad \bmod N\right\} .
$$

Proposition 4.5. Let $\theta \in \mathfrak{H}$ such that $O_{f}=\mathbf{Z}[\theta]$ and $f_{\theta}(X)=X^{2}+B X+$ $C(B, C \in \mathbf{Z})$ the minimal polynomial of $\theta$.

(i) If $h \in A_{0}(N)_{\mathbf{Q}}$ and $h$ is pole-free at $\theta$, then $h(\theta) \in R_{f N}$.

(ii) If $h \in A\left(\Gamma^{0}(N)\right)_{\mathbf{Q}}$, $h$ is pole-free at $\theta$ and $N \mid C$, then $h(\theta) \in R_{f}$.

Proof. Let us use the notation in $\S 2$ of [3]. For a prime number $p$, consider groups

$$
\begin{aligned}
& U_{p}=\left\{\left(\begin{array}{ll}
a & b \\
c & d
\end{array}\right) \in \mathrm{GL}_{2}\left(\mathbf{Z}_{p}\right) \mid c \in N \mathbf{Z}_{p}\right\}, \\
& V_{p}=\left\{\left(\begin{array}{ll}
a & b \\
c & d
\end{array}\right) \in \mathrm{GL}_{2}\left(\mathbf{Z}_{p}\right) \mid b \in N \mathbf{Z}_{p}\right\} .
\end{aligned}
$$


Put $U=\prod_{p} U_{p}, V=\prod_{p} V_{p}$. Then $U$ (resp. $V$ ) is the subgroup of $\prod_{p} \mathrm{GL}_{2}\left(\mathbf{Z}_{p}\right)$ with fixed field $F_{0}=A_{0}(N)_{\mathbf{Q}}\left(\right.$ resp. $\left.F^{0}=A\left(\Gamma^{0}(N)\right)_{\mathbf{Q}}\right)$. Let $O=O_{f}$ be the order of $K$ of conductor $f$. By Theorems 5.5 and 5.7 of [8], we have an exact sequence

$$
1 \longrightarrow O^{*} \longrightarrow \prod_{p} O_{p}^{*} \longrightarrow \operatorname{Gal}\left(K^{a b} / K(j(\theta)) \longrightarrow 1 .\right.
$$

Let $g_{\theta}=\prod_{p}\left(g_{\theta}\right)_{p}: \prod_{p} O_{p}^{*} \longrightarrow \prod_{p} \mathrm{GL}_{2}\left(\mathbf{Z}_{p}\right)$ be the map defined by (4) and (5) in [3]. Since by the definition, for $s, t \in \mathbf{Z}_{p}$,

$$
\left(g_{\theta}\right)_{p}(s \theta+t)=\left(\begin{array}{cc}
t-B s & -C s \\
s & t
\end{array}\right)
$$

we have $\left(g_{\theta}\right)_{p}^{-1}\left(U_{p}\right)=\left(\mathbf{Z}_{p}^{*}+N \mathbf{Z}_{p} \theta\right) \cap O_{p}^{*}=\left(O_{f n}\right)_{p}^{*}$. Therefore we have $g_{\theta}^{-1}(U)=\prod_{p}\left(O_{N f}\right)_{p}^{*}$. If $N \mid C$, then $\left(g_{\theta}\right)_{p}^{-1}\left(V_{p}\right)=O_{p}^{*}$ and $g_{\theta}^{-1}(V)=\prod_{p} O_{p}^{*}$. By class field theory the groups $\prod_{p} O_{p}^{*}$ and $\prod_{p}\left(O_{f N}\right)_{p}^{*}$ correspond to $\operatorname{Gal}\left(K^{a b} / R_{f}\right)$ and $\operatorname{Gal}\left(K^{a b} / R_{f N}\right)$ respectively. By Theorem 2 of [3], we see $R_{f}=K\left(F^{0}(\theta)\right)$ and $R_{f N}=K\left(F_{0}(\theta)\right)$. Therefore we have our assertions.

Corollary 4.6. Let the notation be the same as in Proposition 4.5. Let $\mathfrak{A}=\left[\mathfrak{a}_{1}, \ldots, \mathfrak{a}_{n}\right]$ with $\mathfrak{a}_{i} \in \mathfrak{E}_{1}$ for all $i$. Then we have the followings.

(i) $T_{\mathfrak{A}, F}(\theta) \in R_{f N}$.

(ii) If $N \mid C$, then $T_{\mathfrak{A}, F}(-1 / \theta) \in R_{f}$.

Proof. Since $\Gamma^{0}(N)=S^{-1} \Gamma_{0}(N) S$ with $S=\left(\begin{array}{cc}0 & -1 \\ 1 & 0\end{array}\right), T_{\mathfrak{A}, F} \circ S$ is a modular function with respect to $\Gamma^{0}(N)$. By the result for $t=N$ in (ii) of Proposition [3.4, we know $T_{\mathfrak{A}, F} \in \mathbf{Q}((q))$. Since $B(1,1,1,-1) S^{-1} \in \Gamma_{0}(N)$, we have $T_{\mathfrak{A}, F} \circ S=T_{\mathfrak{A}, F} \circ B(1,1,1,-1)$. By Proposition 3.5, we know $T_{\mathfrak{A}, F} \in A_{0}(N)_{\mathbf{Q}}$ and $T_{\mathfrak{A}, F} \circ S \in A\left(\Gamma^{0}(N)\right)_{\mathbf{Q}}$. Our assertions follow from Proposition 4.5.

Theorem 4.7. Put $\mathfrak{a}_{1}=[2,3,1], \mathfrak{a}_{2}=[2,5,1], \mathfrak{a}_{3}=[1,(N-3) / 2,(N-1) / 2]$ and $\mathfrak{A}=\left[\mathfrak{a}_{1}, \mathfrak{a}_{2}\right]$. Put $F=X_{1}^{m} X_{2}^{n}$ with non-negative integers $m$ and $n$. Let $\theta \in \mathfrak{H}$ such that $O_{f}=\mathbf{Z}[\theta]$ and $f_{\theta}(X)=X^{2}+B X+C(B, C \in \mathbf{Z})$ the minimal polynomial of $\theta$. Then we have followings. 
(i) If $N$ is a prime number and $N>7$, then

$$
R_{f N}=K\left(T_{\mathfrak{a}_{i}}(\theta), T_{\mathfrak{a}_{2}}(\theta)\right)=K\left(T_{\mathfrak{a}_{j}}(\theta), T_{\mathfrak{a}_{1}, \mathfrak{a}_{2}}(\theta)\right),
$$

for $i=1,3$ and $j=1,2,3$. Further if $N \mid C$, then

$$
R_{f}=K\left(T_{\mathfrak{a}_{i}}(-1 / \theta), T_{\mathfrak{a}_{2}}(-1 / \theta)\right)=K\left(T_{\mathfrak{a}_{j}}(-1 / \theta), T_{\mathfrak{a}_{1}, \mathfrak{a}_{2}}(-1 / \theta)\right)
$$

for $i=1,3$ and $j=1,2,3$.

(ii) Assume that $N$ does not divide $5 m+7 n$ (resp. $4(5 m+7 n)$ ) and $N>9$ (resp. 36) in the case $N$ is odd (resp. even). Further assume that $N$ is not divided by 4 in the case $m+n$ is even. Then $R_{f N}=$ $K\left(j(\theta), T_{\mathfrak{A}, F}(\theta)\right)$. Further if $N \mid C$, then $R_{f}=K\left(j(\theta), T_{\mathfrak{A}, F}(-1 / \theta)\right)$

Proof. In the proof of Propositions 4.5 we showed $R_{N f}=K\left(F_{0}(\theta)\right), R_{f}=$ $K\left(F^{0}(\theta)\right)$. Therefore the assertions follow from Propositions 4.2 and Theorem 4.3 .

\section{Class polynomials of $T_{\mathfrak{A}, F}$}

Let $O$ be the order of conductor $f$ of an imaginary quadratic field $K$. Let $D$ be the discriminant and $C(O)$ the (proper) ideal class group of $O$. We denote by $h(D)$ the class number of $O$. Let $\alpha \in K \cap \mathfrak{H}$ and $A X^{2}+B X+C=0$

be the primitive minimal equation with integral coefficients of $\alpha$ over $\mathbf{Q}$. If $D=B^{2}-4 A C$, then we say $\alpha$ is an element of discriminant $D$. We put $I_{\alpha}=[A,(-B+\sqrt{D}) / 2]=\mathbf{Z} A+\mathbf{Z}((-B+\sqrt{D}) / 2)$. Then $I_{\alpha}$ is an ideal of $O$. To compute the singular values of the functions $T_{\mathfrak{A}, F}$, we use an $N$-system for $O$ introduced by Schertz [7].

Definition 5.1. Let $\mathfrak{N}$ be a set of $h(D)$ elements $\alpha_{i} \in K \cap \mathfrak{H}$ of discriminant D. Let $A_{i} X^{2}+B_{i} X+C_{i}=0$ be the primitive integral minimal equation of $\alpha_{i}$ and $I_{\alpha_{i}}=\left[A_{i},\left(-B_{i}+\sqrt{D}\right) / 2\right]$. We say $\mathfrak{N}$ is an $N$-system for $O$ if following conditions are satisfied:

1. $\left(A_{i}, N\right)=1, N \mid C_{i}, B_{i} \equiv B_{j} \bmod 2 N$ for every $i, j$,

2. the set of ideals $\left\{I_{\alpha_{i}}\right\}$ is a transversal of $C(O)$. 
Let $\mathfrak{N}$ be an $N$-system for $O$. Then by Complex multiplication theory, for each $\alpha_{i} \in \mathfrak{N}, j\left(\alpha_{i}\right)$ is an algebraic integer and generates the ring class field $R_{f}$ associated with the order of conductor $f$ and they are conjugate to each other over $\mathbf{Q}$ (see $\S 11 . \mathrm{D}$ of [1]). For singular values $T_{\mathfrak{A}, F}\left(-1 / \alpha_{i}\right)$ we have

Theorem 5.2. Let $N$ be a positive integer such that $\mathfrak{E}_{2}$ is not empty. Put $\mathfrak{A}=\left[\mathfrak{a}_{1}, \ldots, \mathfrak{a}_{n}\right]$ with $\mathfrak{a}_{i} \in \mathfrak{E}_{2}$. Let $\mathfrak{N}=\left\{\alpha_{i}\right\}$ be an $N$-system for $O$. Then we have $T_{\mathfrak{A}, F}\left(-1 / \alpha_{i}\right) \in R_{f}$ and they are conjugate to each other over $K$.

Proof. Since $\Gamma^{0}(N)=S^{-1} \Gamma_{0}(N) S$ with $S=\left(\begin{array}{cc}0 & -1 \\ 1 & 0\end{array}\right), T_{\mathfrak{A}, F} \circ S$ is a modular function with respect to $\Gamma^{0}(N)$. In the proof of Corollary 4.6, we showed $T_{\mathfrak{A}, F} \circ S \in \mathbf{Q}((q))$. Therefore the assertion follows from Theorem 3.1 of [2] and Theorem 3.7 .

For a modular function $g(\tau)$ with respect to $\Gamma_{0}(N)$ and an $N$-system $\mathfrak{N}=\left\{\alpha_{i}\right\}$, we define the class polynomial $H_{\mathfrak{N}}[g](X)$ of $g(\tau)$ by

$$
H_{\mathfrak{N}}[g](X)=\prod_{i=1}^{h(D)}\left(X-g\left(-1 / \alpha_{i}\right)\right) .
$$

The next assertion follows from Theorem 5.2 .

Theorem 5.3. Let $O_{K}$ be the maximal order of $K$. Then the class polynomial $H_{\mathfrak{N}}\left[T_{\mathfrak{A}, F}\right](X) \in O_{K}[X]$.

Let $B$ be an integer such that $B^{2} \equiv D(\bmod 4 N)$. Proposition 3 of [7] shows the existence of $N$-system containing the number $(-B+\sqrt{D}) / 2$. By Lemma 3.1 of [10], we know the class polynomials of a modular function $g$ related to $N$-systems depend only on integers $B$, considered $\bmod 2 N$. We shall fix an $N$-system containing $(-B+\sqrt{D}) / 2$ and denote it by $\mathfrak{N}_{B}$. In the followings, we give some examples of modular equations and class polynomials of the functions $f=T_{\mathfrak{a}}$ or $T_{\mathfrak{a}_{1}, \mathfrak{a}_{2}}$. We shall denote by $H_{B}(X)$ the class polynomial $H_{\mathfrak{N}_{B}}[f]$ in the case the function $f$ is clearly indicated and any confusion can not occur.

Example 5.4. (1) Let $N=7, \mathfrak{a}=[2,3,1]$. Consider the function $T_{\mathfrak{a}}$. Then the modular equation $\Phi(X, j)$ of $T_{\mathfrak{a}}$ is given by

$$
\begin{aligned}
& \Phi(X, j)=X^{8}-36 X^{7}+546 X^{6}-4592 X^{5}+23835 X^{4} \\
& \quad-80304 X^{3}+176050 X^{2}-(j+232500) X+140625+8 j .
\end{aligned}
$$


(a) Let $D=-3, B=5$. Then $h(-3)=1, \mathfrak{N}_{5}=\{(-5+\sqrt{-3}) / 2\}$. We have the class polynomial

$$
H_{5}(X)=X-3(1+\sqrt{-3}) / 2 .
$$

Thus $T_{\mathfrak{a}}((5+\sqrt{-3}) / 14)=3(1+\sqrt{-3}) / 2$. Since $j((1+\sqrt{-3}) / 2)=0$, we have $\Phi(X, 0)=\left(X^{2}-3 X+9\right)\left(X^{2}-11 X+25\right)^{3}$. In fact, $T_{\mathfrak{a}}((5+$ $\sqrt{-3}) / 14)$ is a root of the factor $X^{2}-3 X+9=0$.

(b) Let $D=-59, B=5$. Then we have $h(-59)=3$ and

$$
\begin{aligned}
& \mathfrak{N}_{5}=\{(-5+\sqrt{-59}) / 2,(-5+\sqrt{-59}) / 6,(23+\sqrt{-59}) / 6\} \\
& H_{5}(X)=X^{3}+\frac{15-7 \sqrt{-59}}{2} X^{2}+\frac{-357+45 \sqrt{-59}}{2} X+\frac{717+\sqrt{-59}}{2} .
\end{aligned}
$$

(2) Let $N=13, D=-3, B=7, \mathfrak{a}=[5,3,1]$. Take $\mathfrak{N}_{7}=\{(-7+\sqrt{-3}) / 2\}$. Then the modular equation $\Phi(X, j)$ of $T_{\mathfrak{a}}$ and the value $\left.T_{\mathfrak{a}}(7+\sqrt{-3}) / 26\right)$ are given by

$$
\begin{aligned}
& \Phi(X, j)=\left(X^{2}-9 X+27\right)\left(X^{4}-21 X^{3}+167 X^{2}+-604 X+848\right)^{3}-j(X-7) \\
& T_{\mathfrak{a}}((7+\sqrt{-3}) / 26)=(9+3 \sqrt{-3}) / 2 .
\end{aligned}
$$

Thus in fact $T_{\mathfrak{A}, F}((7+\sqrt{-3}) / 26)$ is a root of $X^{2}-9 X+27=0 D$

(3) Let $N=11, \mathfrak{a}=[2,5,1], D=-7, B=9$. Then $\mathfrak{N}_{9}=\{(-9+\sqrt{-7}) / 2\}$ and we have $T_{\mathfrak{a}}((9+\sqrt{-7}) / 44)=(5+\sqrt{-7}) / 2$ and the modular equation

$$
\begin{aligned}
& \Phi(X, j)=X^{12}-84 X^{11}+2970 X^{10}-57772 X^{9}+680559 X^{8}-5062728 X^{7} \\
& -(22 j-24250028) X^{6}+(561 j-75844824) X^{5}-(2981 j-157525071) X^{4} \\
& \quad-(1177 j+217265444) X^{3}+(26477 j+193124250) X^{2} \\
& \quad-\left(j^{2}+31316 j+101227452\right) X+18 j^{2}+4261 j+24137569 .
\end{aligned}
$$

Since $j((1+\sqrt{-7}) / 2)=-15^{3}$, we have

$$
\begin{aligned}
& \Phi\left(X,-15^{3}\right)=\left(X^{10}-79 X^{9}+2567 X^{8}-44305 X^{7}+438498 X^{6}-2515798 X^{5}\right. \\
& \left.+8237304 X^{4}-16425295 X^{3}+19561039 X^{2}+15914486 X+26848493\right) \\
& \times\left(X^{2}-5 X+8\right) .
\end{aligned}
$$

Therefore, we know $T_{\mathfrak{a}}((9+\sqrt{-7}) / 44)$ is a root of the factor $X^{2}-5 X+8$.

Example 5.5. Let $N=11, \mathfrak{a}=[2,3,1], \mathfrak{b}=[2,3,5]$. Consider the function $T_{\mathfrak{a}, \mathfrak{b}}$. Then we give the coefficients $C_{i}$ of the modular equation $\Phi(X, j)=X^{12}+$ 
$\sum_{i=1}^{12} C_{i} X^{12-i}$ in the table below.

(1) Let $D=-83, B=7$. Then we have $h(-83)=3$ and

$$
\begin{gathered}
\mathfrak{N}_{7}=\{(-7+\sqrt{-83}) / 2,(-7+\sqrt{-83}) / 6,(-29+\sqrt{-83}) / 6\}, \\
H_{7}(X)=X^{3}-(361481+7136 \sqrt{-83}) X^{2}+(57020581+25984608 \sqrt{-83}) X \\
+1683573861-404390656 \sqrt{-83} .
\end{gathered}
$$

(2) Let $D=-39, B=7$. Then we have $h(-39)=4$ and

$$
\begin{gathered}
\mathfrak{N}_{7}=\{(-7+\sqrt{-39}) / 2,(-7+\sqrt{-39}) / 4,(-29+\sqrt{-39}) / 4,(-51+\sqrt{-39}) / 8\}, \\
H_{7}(X)=X^{4}+(-4720+231 \sqrt{-39}) X^{3}+(1491643-329343 \sqrt{-39}) X^{2} / 2 \\
+(-38934427+9970611 \sqrt{-39}) X / 2+64994911-47480958 \sqrt{-39} .
\end{gathered}
$$

\begin{tabular}{|l|l|l|l|}
\hline$i$ & $C_{i}$ & $i$ & \\
\hline 1 & 3660 & 2 & 4754178 \\
\hline 3 & $21879 j+2517699932$ & 4 & $8917579 j+450023862255$ \\
\hline \hline 5 & $10912 j^{2}-21727187108 j+28522470464664$ \\
\hline 6 & $18536243 j^{2}+439266301210 j+155307879800348$ \\
\hline 7 & $1419 j^{3}+6356028822 j^{2}-4268224633178 j-22718073239498472$ \\
\hline 8 & $1663761 j^{3}+70427463557 j^{2}-129554423289764 j+430444117263292143$ \\
\hline 9 & $66 j^{4}-100966360 j^{3}+544875974962 j^{2}+1322596244939332 j-4047340123195216100$ \\
\hline 10 & $82687 j^{4}-2985616392 j^{3}+3765768493971 j^{2}-9777105305922130 j+21981914597781276930$ \\
\hline 11 & $j^{5}+1956838 j^{4}-26707875453 j^{3}+49826805469384 j^{2}+21725643544520963 j$ \\
\hline 12 & -67067772106836815988 \\
\hline & $\begin{array}{l}\mid 229 j^{5}+29053078 j^{4}-41072974661 j^{3}-92728235099098 j^{2}+68572479313531217 j \\
+93554961663154376449\end{array}$ \\
\hline
\end{tabular}

Example 5.6. Let $N=17, \mathfrak{a}=[1,2,7], \mathfrak{b}=[1,2,3]$. Consider the function $T_{\mathfrak{a}, \mathfrak{b}}$. Let $D=-84, B=8$. Then we have $h(-84)=4$ and

$$
\begin{aligned}
& \mathfrak{N}_{8}=\{-8+\sqrt{-21},(43+\sqrt{-21}) / 11,(-8+\sqrt{-21}) / 5,(9+\sqrt{-21}) / 3\} \\
& H_{8}(X)=X^{4}+(779-157 \sqrt{-21}) X^{3}+(-41194-175 \sqrt{-21}) X^{2} \\
& \quad+(690208+81256 \sqrt{-21}) X-3246464-566976 \sqrt{-21}
\end{aligned}
$$

\section{References}

[1] D.Cox, Primes of the form $x^{2}+n y^{2}$, A Wiley-Interscience Publication, John Wiley and Sons,Inc.,1989 
[2] A.Enge and R.Schertz, Constructing elliptic curves over finite fields using double eta-quotients, J.Théor.Nombres Bordeaux 16 (2004), 555568.

[3] A.Gee, Class invariants by Shimura's reciprocity law, J.Théor.Nombres Bordeaux 11 (1990),45-72.

[4] N.Ishida and N.Ishii, Generators and defining equation of the modular function field of the group $\Gamma_{1}(N)$, Acta Arith. 101.4 (2002),303-320.

[5] N.Ishii, Rational expression for $J$-invariant function in terms of generators of modular function fields, Int.Math. Forum 2 (2007) no. 38, 1877 -1894 .

[6] S.Lang, Elliptic Functions, Springer-verlag,1987.

[7] R.Schertz, Weber's class invariants revisited, J.Théor. Nombres de Bordeaux 14(1) (2002), 325-343.

[8] G.Shimura, Introduction to the Arithmetic Theory of Automorphic Functions, Iwanami-Shoten and Princeton University Press,1971.

[9] J.Silverman, The Arithmetic of Elliptic curves, Springer-verlag,1986.

[10] S.Yoshimura,A.Comuta and N.Ishii, $N$-systems,class polynomials of double eta-quotients and singular values of $j$-invariant function, Int.Math.Forum 4 (2009) no.8,367-376.

Faculty of Liberal arts and Sciences

Osaka Prefecture University

1-1 Gakuen-cho, Naka-ku Sakai

Osaka, 599-8531 Japan

e-mail: ishii@las.osakafu-u.ac.jp
Graduate School of Science

Osaka Prefecture University

1-1 Gakuen-cho, Naka-ku Sakai

Osaka, 599-8531 Japan 